\title{
Resolution of Splenic Injury After Nonoperative Management
}

\author{
By Thomas Pranikoff, Ronald B. Hirschl, Alan E. Schlesinger, Theodore Z. Polley, and Arnold G. Coran \\ Detroit, Michigan and Ann Arbor, Michigan
}

\begin{abstract}
- Numerous studies have demonstrated success with nonoperative management of splenic injuries in pediatric patients. However, the resolution of the splenic injury has not been previously evaluated. The records of $\mathbf{5 0}$ pediatric patients with splenic injuries from blunt trauma treated nonoperatively between 1984 to 1992 were reviewed retrospectively. Abdominal computed tomography (CT) was performed at the time of injury and 6 weeks postinjury in 25 patients. These scans were reviewed and categorized by a modification of a previously reported grading system for parenchymal injury. All patients had healing of the splenic injuries, with complete resolution of the healing process observed at 6 weeks postinjury in $44 \%$. Even those with shattered spleens ( $n=6$ ) had consistent improvement in splenic architecture, with resolution of fractures and/or contusions and return of splenic perfusion. Ten (77\%) of 13 grade 1 and 2 injuries were completely resolved by the 6-week follow-up examination, whereas only one $(8 \%)$ of 12 grade 3 to 5 injuries showed radiological resolution of splenic injuries. None of the 25 follow-up CT scans affected clinical decision-making or led to a deviation from the established protocol, which included a 3-month period of reduced activity. All 50 patients did well, without evidence of morbidity, mortality, or complications after return to full activity 3 months postinjury. These CT scan results and clinical data show (1) radiological resolution of splenic injury, with return toward a normal appearance by 6 weeks postinjury, (2) uniform success of nonoperative management of splenic injury in children, (3) that follow-up CT scans may be used to indicate earlier return to full activity in most cases of grade 1 and 2 splenic injuries, but otherwise they do not appear to contribute to the clinical management of the child after nonoperative treatment of splenic trauma. Copyright $\odot 1994$ by W.B. Saunders Company
\end{abstract}

INDEX WORDS: Splenic injury, nonoperative management.

$\mathbf{T}$ HE MANAGEMENT of splenic injuries has evolved since the 1940s when Wansborough, from the Hospital for Sick Children in Toronto, reviewed the necropsy of a child with a suspected previous splenic injury, and noted a well-healed complete transection of the spleen into two segments on two separate pedicles. ${ }^{1} \mathrm{He}$ suggested that splenec-

From the Division of Pediatric Surgery, Henry Ford Hospital, Detroit, MI, and the Sections of Pediatric Surgery and Pediatric Radiology, C.S. Mott Children's Hospital and the University of Michigan, Ann Arbor, MI.

Date accepted: November 11, 1993.

Presented at the 26th Annual Meeting of the Pacific Association of Pediatric Surgeons, Cairns, Australia, May 9-14, 1993.

Address reprint requests to Ronald B. Hirschl, MD, L2110 Maternal Child Health Center, 1500 E Medical Center Dr, Ann Arbor, MI 48109-0245.

Copyright (1) 1994 by W.B. Saunders Company

0022-3468/94/2910-0018\$03.00/0 tomy was not always necessary for hemodynamically stable patients with splenic injuries. ${ }^{2}$ Since that time, several studies from that institution have supported the nonoperative management of splenic injuries in children. ${ }^{1-7}$ Nonoperative management of splenic injuries has become the standard of care in the treatment of pediatric trauma victims.

In 1989, we reported our experience with nonoperative management of splenic injury in children and adults. ${ }^{8}$ Since then, we have routinely managed hemodynamically stable children with splenic injuries nonoperatively, with complete success. However, we have been concerned about the time it takes for the splenic injury to heal because this will determine how long the child will be on restricted activity. In addition, we questioned whether there was any value in the routine performance of a follow-up abdominal computed tomography (CT) scan to document splenic healing. This study was undertaken to answer these questions.

\section{MATERIALS AND METHODS}

The hospital records of 59 children (mean age, $11 \pm 5$ years; range, 0 to 18 years) admitted to Henry Ford Hospital and the University of Michigan Medical Center with splenic injuries sustained during blunt trauma, between 1984 and 1992, were rcvicwed rctrospcctivcly. Nine children (15\%) with splenic injuries who were hemodynamically unstable underwent emergency laparotomy. Seven of them required splenectomy, and two underwent splenorrhaphy. These patients were not included further in this study.

The remaining 50 children were treated nonoperatively. The abdominal CT scans, both at the time of injury and 6 weeks postinjury, were available for 25 of these patients, who comprise the study group. The CT scans were reviewed, and the splenic injury was categorized, by a modification of a previously reported grading system of parenchymal injury, as follows: grade 0 , no radiological abnormality of the spleen; grade 1, single laceration of the spleen; grades 2 through 4 include multiple fractures or contusions of the spleen involving less than $25 \%, 25 \%$ to $50 \%$, or more than $50 \%$, respectively; and grade 5 , the shattered spleen. ${ }^{9}$

Patients admitted for nonoperative management of their splenic injury had follow-up with an established protocol that required initial hemodynamic stability. All patients were closely monitored in the pediatric intensive care unit, on strict bed rest, for at least 24 hours. Frequent physical examinations, close hemodynamic monitoring, and serial hematocrit measurements were performed to detect ongoing hemorrhage. Blood transfusions were administered based on the hematocrit level and volume status. Indications for laparotomy included evidence of continuing blood loss (tachycardia, hypotension, oliguria, and/or blood transfusion requirements exceeding $50 \mathrm{~mL} / \mathrm{kg} / 24 \mathrm{~h}$ ). Patients who remained stable for 24 hours wcre transferred to the surgical ward and were managed during bed rest for a total of 7 days. Their diet was advanced as tolerated. After the 7 days, resolution of abdominal pain and tenderness along with tolerance of a regular diet was considered an 
Table 1. Splenic Injury Grading Based on CT Scan at Time of Admission and 6 Weeks Postinjury ( $n=25$ )

\begin{tabular}{ccc}
\hline Grade & Admission & 6-wk Follow-Up \\
\hline 0 & 0 & 11 \\
1 & 4 & 1 \\
2 & 9 & 9 \\
3 & 5 & 4 \\
4 & 1 & 0 \\
5 & 6 & 0 \\
\hline
\end{tabular}

indication for discharge for patients who did not have other conditions necessitating continued hospitalization. Activities were restricted for 3 months after discharge, after which most patients were allowed to resume a full range of physical activities.

\section{RESULTS}

Fifty patients were treated nonoperatively. None required subsequent or delayed laparotomy. Twentyfive of the patients had an initial and a 6-week follow-up CT scan. The injury grading distribution appears in Table 1. At the time of admission, four patients had grade 1 injuries, nine had grade 2, five had grade 3 , one had grade 4 , and six had grade 5 . Six weeks postinjury, the CT scans showed evidence of partial or complete splenic injury resolution in all patients. A grade 0 injury (no abnormality) was cvident in 11 paticnts, grade 1 in one, grade 2 in nine, and grade 3 in four. No grade 4 or 5 injury was observed 6 weeks postinjury. The course of splenic healing in each patient is demonstrated in Fig 1. Eleven patients $(44 \%)$ had complete resolution of abnormalities. Ten (77\%) of 13 grade 1 and 2 injuries and one $(8 \%$ ) of 12 grade 3,4 , and 5 injuries were completely healed by the 6 week follow-up evalua- tion. Two grade 2 and one grade 1 case had the same injury grade at the follow-up evaluation. Although they did not meet the criteria necessary for advancement to a lower grade, all three had radiological evidence of healing and partial resolution of the splenic injury.

Evidence of injury on the initial CT scan included parenchymal fractures, hematoma formation, nonperfused splenic tissue, and free intraabdominal fluid. The CT scan 6 weeks later showed improvement or resolution of almost all the initial findings. This was true even for the six cases of shattered spleens. The initial radiological presentation of these shattered spleens, as seen in Fig 2, demonstrated massive contusion with multiple diffuse fractures throughout the splenic parenchyma. Poorly perfused areas of the spleen were observed, and free intraabdominal fluid was noted. The 6-week follow-up CT scan showed obvious healing, with disappearance of the majority of the previously observed splenic fractures and reperfusion of previously nonperfused regions of the spleen.

All 50 patients did well. There were no delayed sequelae such as reinjury or bleeding. Review of the medical records did not show any evidence that management had been altered by the results of any of the 25 follow-up CT scans. Specifically, the period of activity restriction was not extended beyond the 3-month routine for any patient, based on the follow-up CT scan findings. No development of abscess, hematoma, pseudocyst, pseudoaneurysm, or other anatomic abnormalities was observed that led to a change in subsequent clinical management.
Fig 1. Progression of CT scan injury grade for each patient, from admission (solid bars) to the 6-week follow-up evaluation (hatched bars).

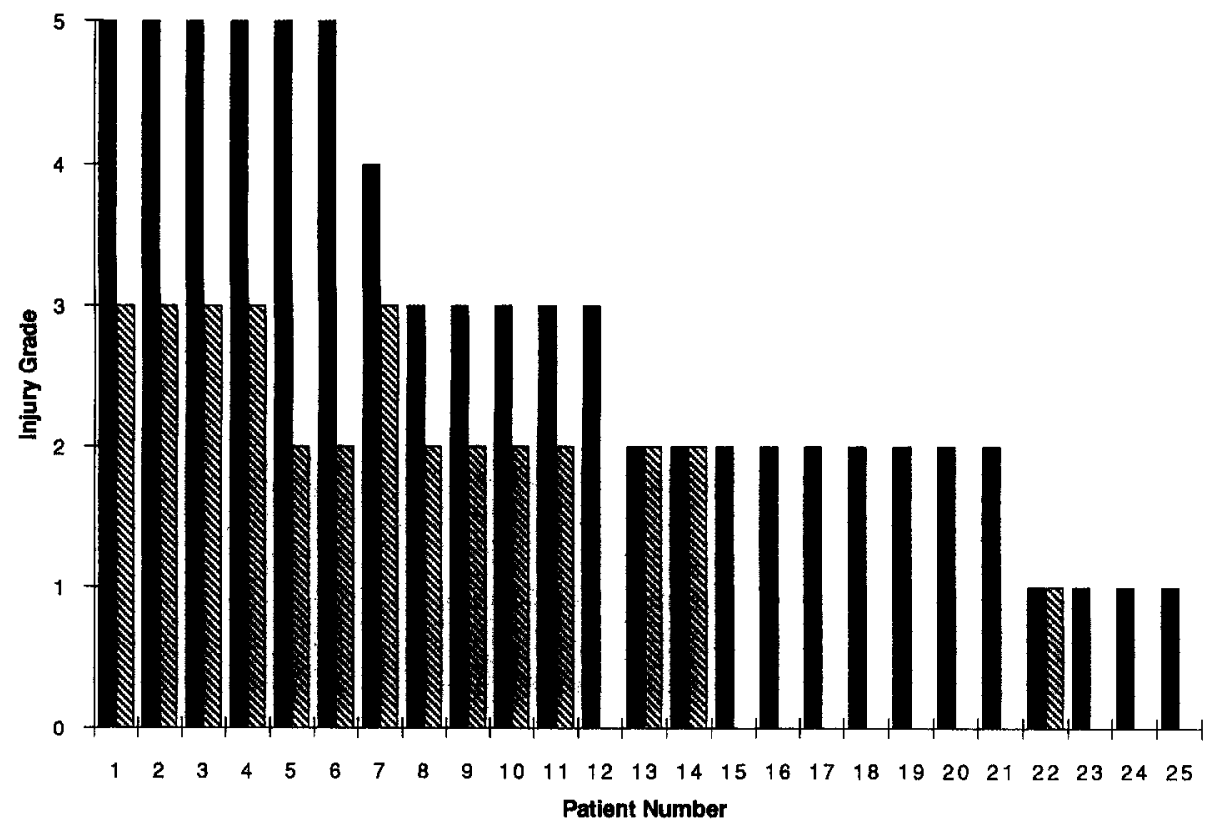



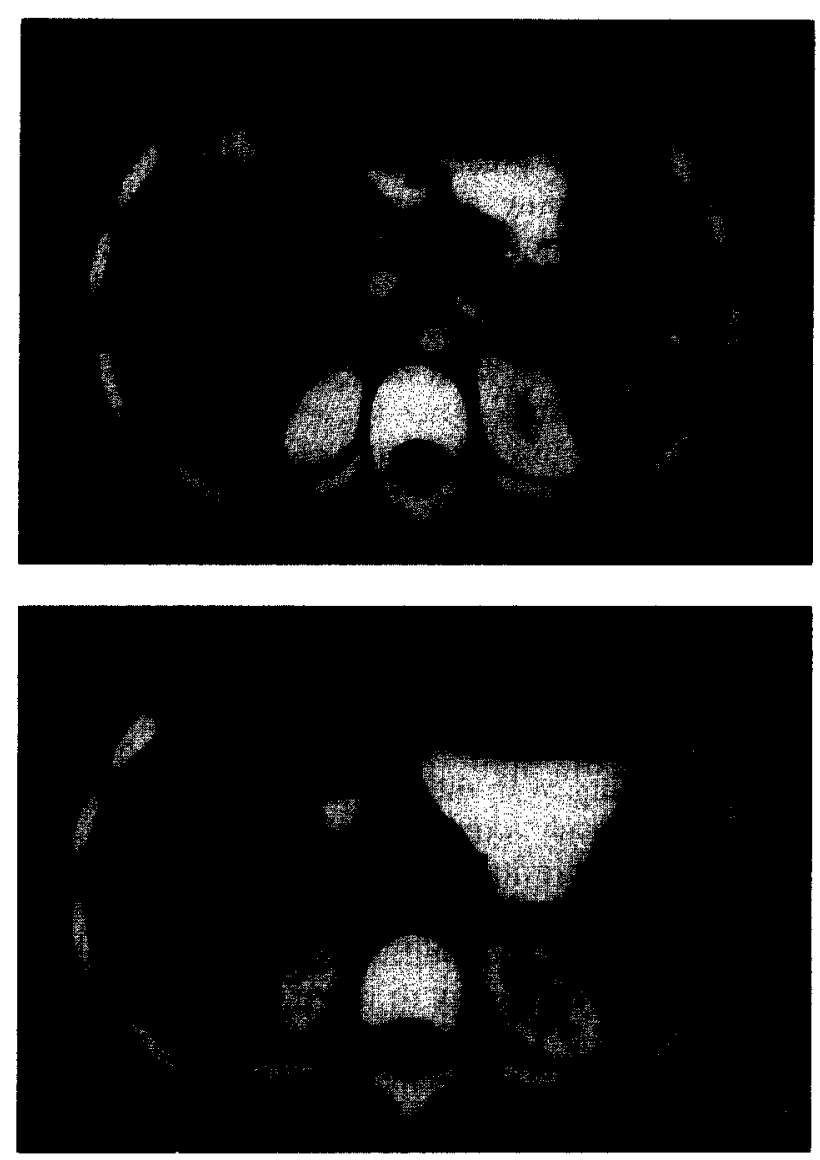

Fig 2. CT scan of a shattered spleen (grade 5 injury) at the initial evaluation. (A) Free intraperitoneal fluid is observed around the liver. (B) Six-week follow-up assessment showed marked normalization of splenic architecture and improvement of parenchymal perfusion (grade 2).

\section{DISCUSSION}

Upadhyaya and Simpson reported the first series of splenic injuries in children managed without operation, from the Hospital for Sick Children in Toronto, in $1968 .^{3}$ They described 12 children with signs and symptoms consistent with splenic rupture who were successfully treated nonoperatively. From this experience, they suggested that spleen injuries could be successfully managed without operation. Subsequent studies have proven this to be a successful management strategy. ${ }^{1-3,5-7}$ None of the children with splenic injuries in these studies required operation after remaining hemodynamically stable for 24 hours after injury. In the most recent study from the Toronto group, Pearl et al noted a continuous increase in the splenic salvage rate, with $87 \%$ of patients treated nonoperatively between 1981 and 1986. Stable patients with splenic injuries at their institution are now admitted directly to the surgical ward and remain hospitalized for only 7 days. $^{2}$
Previously we demonstrated that splenic salvage is enhanced by nonoperative management of splenic trauma and that there is an associated reduction in blood transfusion requirements. ${ }^{8}$ Therefore, the nonoperative management of splenic injury allows splenic preservation and thereby eliminates the risk of overwhelming postsplenectomy infection in the child. ${ }^{10-13}$ Although the nonoperative approach to splenic injury is clearly successful, there were no reports of a CT scan being used in the follow-up course of children. The protocol for nonoperative management of children with splenic injury at our two institutions included an initial CT scan (at the time of presentation) and a follow-up study 6 weeks postinjury. Thus, we were able to evaluate the 25 patients whose CT scans were available for review and to assess splenic healing during the 6 weeks after injury. There was at least partial splenic healing in all patients, $11(44 \%)$ of whom had complete healing during the 6-week period; some of these injuries had been grade 2 or 3 . Even all the shattered spleens had substantial resolution of injury, with progression toward normal architecture and return of parenchymal perfusion.

None of the 25 follow-up CT scans in this series resulted in a change in the clinical management. Most pediatric trauma centers using the nonoperative approach to splenic trauma employ routine CT scan evaluation of the spleen approximately 4 to 8 weeks after injury. However, there has been no previous assessment of the value of this practice. Although relatively minor, CT scan evaluation has its own risks, which include serious contrast reactions, the potential need for sedation and/or general anesthesia, and exposure to ionizing radiation. In addition, the cost of each scan ranges from $\$ 1,000$ to $\$ 2,000$. The data from this review suggest that routine follow-up CT scan evaluation may not be necessary in the asymptomatic patient after nonoperative management of splenic injury.

Empirically, previous investigators have recommended a reduced activity level for 2 to 3 months after nonoperative management of splenic trauma. ${ }^{2}$ We chose to limit activity for 3 months after injury, by protocol. Although approximately half the injured spleens had radiological evidence of complete healing 6 weeks postinjury, we are unable to estimate the amount of time over which healing occurred in the remainder. However, since half the spleens, including some grade 1 and 2 injuries, were not completely healed 6 weeks after injury, a designated period of inactivity for 3 months appears to be reasonable. Among the 50 patients managed with 3 months of 
reduced activity, no subsequent complications were observed.

Based on our clinical observations and the CT scan data, we suggest the following management alternatives: (1) limitation of activity for all patients for 3 months, without follow-up CT scan evaluation, or (2) patients with grade 1 or 2 injuries may be evaluated by CT scan 6 weeks postinjury. If resolution is noted, full activities may be resumed. If persistent injury is observed, activities should remain restricted for the full 3 months. No further CT scan follow-up is indicated. This allows for $77 \%$ of all patients with grade 1 and 2 splenic injuries to return to full activity at an earlier time. Because only $8 \%$ of grade 3 to 5 injuries are fully healed by 6 weeks postinjury, we suggest that activity be reduced for the full 3 months, without follow-up CT scan evaluation.

Concern about adverse sequelae with nonoperative management of splenic injuries has been expressed. In our study, we did not find any adverse sequelae such as abscess, pseudocyst, pseudoaneurysm, or splenic infarction after nonoperative management. However, development of a splenic pseudocyst after nonoperative management, although rare and of questionable clinical significance, has been reported. The nced to perform routine CT evaluation after nonoperative management of splenic injuries, to identify patients with such an abnormality, was not addressed in this study.

In conclusion, nonoperative management of splenic injury in children in this series was uniformly successful. Radiological evaluation showed evidence of total resolution or marked improvement of the disruption in splenic architecture 6 weeks postinjury. Follow-up CT scans can be used to indicate earlier return to full activity for most patients with grade 1 and 2 splenic injuries, but otherwise they do not appear to contribute to the clinical management of the child after nonoperative treatment of splenic trauma.

\section{REFERENCES}

1. Douglas GJ, Simpson JS: The conservative management of splenic trauma. J Pediatr Surg 6:565-570, 1971

2. Pearl RH, Wesson DE, Spence LJ, et al: Splenic injury: 5-year update with improved results and changing criteria for conservative management. J Pediatr Surg 24:428-431, 1989

3. Upadhyaya P, Simpson JS: Splenic trauma in children. Surg Gynecol Obstet 126:781-790, 1968

4. Ein SH, Shandling B, Simpson JS, et al: The morbidity and mortality of splenectomy in childhood. Ann Surg 185:307-310, 1977

5. Ein SH, Shandling B, Simpson JS, et al: Nonoperative management of traumatized spleen in children: How and why. J Pediatr Surg 13:117-119, 1978

6. Howman-Giles R, Gilday DL, Venugopal S, et al: Splenic
trauma-Nonoperative management and long-term follow-up by scintiscan. J Pediatr Surg 13:121-126, 1978

7. Wesson DE, Filler RM, Ein SH, et al: Ruptured spleenWhen to operate? J Pediatr Surg 16:324-326, 1981

8. Delius RE, Frankel W, Coran AG: A comparison between operative and nonoperative management of blunt injuries to the liver and spleen in adult and pediatric patients. Surgery 106:188193, 1989

9. Resciniti A, Fink MP, Raptopoulos V, et al: Nonoperative treatment of adult splenic trauma: Development of a computed tomographic scoring system that detects appropriate candidates for expectant management. J Trauma 128:828-831, 1988 